\title{
An Optimized Forecasting Approach Based on Grey Theory and Cuckoo Search Algorithm: A Case Study for Electricity Consumption in New South Wales
}

\author{
Ping Jiang, ${ }^{1}$ Qingping Zhou, ${ }^{2}$ Haiyan Jiang, ${ }^{2}$ and Yao Dong ${ }^{3}$ \\ ${ }^{1}$ School of Statistics, Dongbei University of Finance and Economics, Dalian 116025, China \\ ${ }^{2}$ School of Mathematics and Statistics, Lanzhou University, Lanzhou 730000, China \\ ${ }^{3}$ Department of Statistics, Florida State University, Tallahassee, FL 32310, USA \\ Correspondence should be addressed to Qingping Zhou; zhouqp12@lzu.edu.cn
}

Received 17 March 2014; Accepted 18 April 2014; Published 3 June 2014

Academic Editor: Fuding Xie

Copyright ( 2014 Ping Jiang et al. This is an open access article distributed under the Creative Commons Attribution License, which permits unrestricted use, distribution, and reproduction in any medium, provided the original work is properly cited.

\begin{abstract}
With rapid economic growth, electricity demand is clearly increasing. It is difficult to store electricity for future use; thus, the electricity demand forecast, especially the electricity consumption forecast, is crucial for planning and operating a power system. Due to various unstable factors, it is challenging to forecast electricity consumption. Therefore, it is necessary to establish new models for accurate forecasts. This study proposes a hybrid model, which includes data selection, an abnormality analysis, a feasibility test, and an optimized grey model to forecast electricity consumption. First, the original electricity consumption data are selected to construct different schemes (Scheme 1: short-term selection and Scheme 2: long-term selection); next, the iterative algorithm (IA) and cuckoo search algorithm (CS) are employed to select the best parameter of GM(1,1). The forecasted day is then divided into several smooth parts because the grey model is highly accurate in the smooth rise and drop phases; thus, the best scheme for each part is determined using the grey correlation coefficient. Finally, the experimental results indicate that the GM(1,1) optimized using CS has the highest forecasting accuracy compared with the GM(1,1) and the GM(1,1) optimized using the IA and the autoregressive integrated moving average (ARIMA) model.
\end{abstract}

\section{Introduction}

Electricity-supply planning requires optimizing decisions on hourly consumption for the next day and effective power system. Correspondingly, the power system operator is responsible for scheduling generators and balancing the power supply and consumption [1]. Electricity consumption reflects the degree of economic development in a country, and much evidence supports a causal relationship between economic growth and energy consumption [2-10]. To promote economic growth and fulfill power requirements in the future, electricity consumption forecasting has become a challenging task for electric utilities. Accurate electricity consumption forecasts can aid power generators in scheduling their power station operations to match the installed capacity [11]. Moreover, accurate forecasts are also a prerequisite for decision makers to develop an optimal strategy that includes risk reduction and improving the economic and social benefits. Improper and inaccurate forecasts will lead to electricity shortage, energy resource waste, and grid collapse [12]. Therefore, forecast electricity consumption to manage a power system is significant. Electricity consumption shows typical nonlinear fluctuation and random behaviors, which is influenced by various unstable factors, including climate change and the social environment. Climate changes involve a change in season and temperature, among other considerations, and the social environment refers to law, policy, technical progress, holidays, and the day of the week, among other concerns [13]. On the other hand, with the increasing complexity of power systems, many uncertain factors could influence electricity consumption. Consequently, it is crucial to accurately forecast electricity consumption.

A variety of methods have been proposed to forecast electricity consumption [14,15], electricity load, and electricity 
prices over the last few decades, including linear regression analysis, time series methods, and artificial intelligence. For example, Antoch et al. [16] applied a functional linear regression model to analyze electricity consumption data sets in Sardinia. Mohamed and Bodger [17] used a multiple linear regression model to forecast electricity demand in New Zealand, in which the dependent variable was electricity consumption and the independent variables were the gross domestic product, average price of electricity, and population of New Zealand. However, a linear regression analysis is limited by a number of assumptions, such as weak exogeneity, error independence, and a lack of predictor multicollinearity [18]. After eliminating data noise through the empirical model decomposition method (EMD), Dong et al. [19] first employed the definite season index method and ARIMA model to forecast electricity prices in New South Wales of Australia. Ohtsuka et al. [20] presented a spatial autoregressive ARMA(1,1) model to forecast regional electricity consumption in Japan. Zhao et al. [11] proposed a residual modification model to improve forecasting precision for a seasonal ARIMA model in China's Northwest Power Grid. In general, time series models only consider the data, not other relative factors, and require high quantities of sample data with a good statistical distribution. In addition, artificial neural networks with the back propagation-learning algorithm have attracted much attention [21-23], but artificial intelligence approaches often suffer from low converging rates, difficulty in parameter selection, and overfitting [24, 25].

The sample size is a key element that affects the forecast performance, and it limits forecasting applicability under certain situations; although it is available to obtain a sufficient historical data set, it often differs from the growth of actual electricity consumption considerably. Electricity consumption data typically exhibit an increasing fluctuation trend, which is unsuitable for autoregressive moving average, exponential smoothing, and multiple linear regression models. Therefore, new forecasting models must be created for limited samples and uncertain conditions [12]. Considering these problems, grey-based forecasting models have recently garnered much attention because they are especially suitable for forecasting using uncertain and insufficient information [26].

Grey system theory was pioneered by Ju-Long [27] and identifies hidden original data by transforming irregular original data into strong regular data through an accumulating generation operator (AGO) [28]. The $\operatorname{GM}(1,1)$ is the main grey theory forecasting model with good short-term forecasting accuracy. Due to the few samples required and its fast calculations, it is successfully used in engineering, technology, industrial and agricultural production, economics, and many other fields [29-34]. However, for practical GM(1,1) applications, the forecasting accuracy may decrease when the original data show an increasing trend [35] or when the data samples rapidly mutate [13].

In this paper, after integrating the original data with different selections, feasibility testing, and selecting the best scheme for different forecasting segments, a parameteroptimized $\operatorname{GM}(1,1)$ is proposed for forecasting electricity consumption. At first, the original electricity consumption series were used to construct different schemes from the short- and long-term aspects. The electricity demand data at a given hour on different days varies similarly; thus, we used data from the same hour on different weeks. Second, through selecting the appropriate original data, an abnormality analysis and feasibility test can be used to improve the forecast accuracy. Third, optimization algorithms were applied to select the best parameter $\alpha$ in the $\operatorname{GM}(1,1)$. Based on fast convergence and generating a good optimization solution, an iterative algorithm and the cuckoo search algorithm can be employed [36]. Once the best parameter is obtained using optimization methods, the GM(1,1) should perform well [37]. We divided the forecasted day into several smooth parts using certain criteria because the $\operatorname{GM}(1,1)$ is highly accurate in the smooth rise and drop phases [27]. We determined the best scheme for each part using the grey correlation coefficient between the actual and forecasted consumptions. Finally, the scheme with the largest grey correlation coefficient was considered the forecasting scheme, and by combining the best forecasts the final forecasts are obtained.

This paper is organized as follows. Section 3 introduces the $\operatorname{GM}(1,1)$ and two parameter optimization algorithms, including an iterative algorithm and a cuckoo search algorithm. Section 4 describes the preprocessing procedure and transformation of available data for a successful $\operatorname{GM}(1,1)$. Section 5 discusses the simulation procedure for the proposed method, experimental results, and error analyses. Finally, Section 6 concludes this paper.

\section{Our Contributions}

We propose an effective hybrid method, the CSGM, to forecast electricity consumption in NSW. Based on the inherent characteristics of $\mathrm{GM}(1,1)$, a series of suitable concepts, which include data selection, an abnormality analysis, a feasibility test, and optimized algorithms, were used to improve forecasting accuracy. A case study shows that CSGM performs better than the classic $\operatorname{GM}(1,1)$, the $\operatorname{GM}(1,1)$ optimized using IA and the ARIMA model. Finally, we analyzed the forecasting errors based on statistical theory, which showed that the ARIMA electricity consumption forecasting model yielded a significant result with a small average error but with a high error at certain time-points; thus, ARIMA is not a suitable consumption forecasting model of electricity consumption in NSW.

\section{Materials and Methods}

In this section, we first introduce the classic GM(1,1) model; next, two types of optimized algorithms are used to select the optimal parameter in the $\mathrm{GM}(1,1)$ model.

3.1. The $\operatorname{GM}(1,1)$ Model. The $\mathrm{GM}(1,1)$ includes a set of differential equations with structures that vary with time rather than a single, general first-order differential equation. Although it is not necessary to use all of the data from the original time series to construct the $\operatorname{GM}(1,1)$, the potency of the series data must be more than four. The procedures for 
establishing and constructing a general GM(1,1) are described below.

The $\operatorname{GM}(1,1)$ is a first-order and single-variable grey model that consists of a grey differential equation.

Step 1. The original nonnegative data series $X^{(0)}$ with $m$ samples denotes the electricity consumption in NWS, which is expressed as follows:

$$
X^{(0)}=\left(x^{(0)}(1), x^{(0)}(2), \ldots, x^{(0)}(m)\right),
$$

where the superscript (0) represents the original series and $x^{(0)}(k)$ represents the electricity demand of the data at the time index $k$ for $k=1,2, \ldots, m$.

Step 2. Obtain the 1-AGO (one-time accumulating generation operation) sequence $X^{(1)}$ by imposing the first-order accumulating generator operator to $X^{(0)}$, which monotonically increases and is expressed as follows:

$$
X^{(1)}=\left(x^{(1)}(1), x^{(1)}(2), \ldots, x^{(1)}(m)\right),
$$

where $x^{(1)}(k)=\sum_{i=1}^{k} x^{(0)}(i)$, as $k=1,2, \ldots, m$.

Step 3. The general GM(1,1) is described by the following grey differential equation:

$$
x^{(0)}(k)+a \cdot z^{(1)}(k)=b, \quad k=2,3, \ldots, m,
$$

where $a$ is the grey developmental coefficient and $b$ is the grey control parameter. Thus,

$$
z^{(1)}(k)=(1-\alpha) x^{(1)}(k)+\alpha x^{(1)}(k-1), \quad k=2,3, \ldots, m,
$$

where $z^{(1)}(k)$ is referred to as the background value of the grey derivative and $\alpha$ is the background value production coefficient that must be optimized for the interval $[0,1]$. The $\operatorname{GM}(1,1)$ with $\alpha$ equals 0.5 and is referred to as $\operatorname{GM}(1,1)$.

Step 4. Using the least-square estimation method, the approximate values for $a$ and $b$ can be estimated as follows:

$$
\left[\begin{array}{l}
a \\
b
\end{array}\right]=\left(B^{T} B\right)^{-1} B^{T} Y
$$

where

$$
B=\left[\begin{array}{ccc}
-z^{(1)}(2) & 1 \\
-z^{(1)}(3) & 1 \\
\vdots & \vdots \\
-z^{(1)}(m) & 1
\end{array}\right], \quad Y=\left[\begin{array}{c}
x^{(0)}(2) \\
x^{(0)}(3) \\
\vdots \\
x^{(0)}(m)
\end{array}\right]
$$

Step 5. The solution to (3) can be determined after substituting the obtained parameters $a$ and $b$ into (3). $X^{(1)}$ at time $k$ is described as follows:

$$
\widehat{x}^{(1)}(k)=\left(x^{(0)}(1)-\frac{b}{a}\right) \cdot e^{-a(k-1)}+\frac{b}{a}, \quad k=1,2, \ldots, m .
$$

Step 6. To obtain the predicted values for $\widehat{X}^{(0)}$, the IAGO (inverse accumulated generating operation) is used to establish the following grey model:

$$
\begin{gathered}
\widehat{x}^{(0)}(1)=x^{(0)}(1), \quad k=1, \\
\widehat{x}^{(0)}(k)=\widehat{x}^{(1)}(k)-\widehat{x}^{(1)}(k-1), \quad k=2,3, \ldots, m .
\end{gathered}
$$

Equation (8) is then equivalent to the following:

$$
\begin{array}{r}
\widehat{x}^{(0)}(k)=\left(x^{(0)}(1)-\frac{b}{a}\right) \cdot e^{-a(k-1)} \cdot\left(1-e^{-a}\right), \\
k=1,2, \ldots, m .
\end{array}
$$

From the above introduction, the general GM(1,1) contains the adjustable parameter that must be determined from the available experimental data. Therefore, how this parameter is optimized is important when applying the general $\mathrm{GM}(1,1)$.

3.2. Parameter Optimization Using an Iterative Algorithm (IAGM). Equation (5) shows that the parameters $a$ and $b$ are related to the raw data series $X^{(0)}$ and production coefficient $\alpha$, which are background values. $X^{(0)}$ are the historical data; thus, the controllable parameter is $\alpha$. The traditional background value in the general $\operatorname{GM}(1,1)$ typically takes the following calculation equation, $\alpha=0.5$ :

$$
z^{(1)}(k)=\frac{1}{2}\left(x^{(1)}(k)+x^{(1)}(k-1)\right) .
$$

Zhuan [38] proved that the accurate calculation equation for the background value $z^{(1)}(k)$ defined in (4) should satisfy the relationship between the parameter $\alpha$ and the developing coefficient $a$ as follows:

$$
\alpha=\frac{1}{a}-\frac{1}{e^{a}-1} .
$$

Chang et al. [39] demonstrated that the model's forecasting accuracy can be improved by optimizing the parameter $\alpha$. To improve the accuracy of $\operatorname{GM}(1,1)$, this paper uses an iterative algorithm [37]; the parameter $\alpha$ is optimized for $\mathrm{GM}(1,1)$ as follows.

Step 1. Let $\alpha=0.5$. The parameters $a$ and $b$ are determined using the least-square estimation method according to (5).

Step 2. Substitute the obtained $a$ into (11); then, recalculate $\alpha$, which is denoted by $\alpha(n+1), n=1,2, \ldots$. Given the arbitrarily small positive integer $\varepsilon, \alpha(n+1)$ and $\alpha(n)$ are compared. If $|\alpha(n+1)-\alpha(n)|>\varepsilon$, go to Step 1 and substitute $\alpha(n+1)$ into (4) to calculate the background value $z^{(1)}(k+1)$. Next, GM(1,1) is reconstructed, and the forecasting process is reapplied. If $|\alpha(n+1)-\alpha(n)|<\varepsilon$, stop the iteration cycle and go to Step 3.

Step 3. The $\mathrm{GM}(1,1)$ forecasting model is implemented in accordance with (7). By performing the IAGO using $\widehat{x}^{(1)}(k)$, the forecasting value $\hat{x}^{(0)}(k)$ can be obtained as shown in (9). 
3.3. Parameter Optimization Using the Cuckoo Search Algorithm (CSGM). The cuckoo search algorithm (CS) is a new optimization method with an evolutionary process. CS begins with an initial cuckoo population with different societies, which are composed of two types: mature cuckoos and their eggs. The basic CS is defined by the effort to survive among cuckoos. Certain cuckoos or their eggs die during the survival competition. The surviving cuckoo societies immigrate to a better environment and begin reproducing and laying eggs.

To solve an optimization problem using CS, the problem variable values can be regarded as an array, which can be interpreted as a habitat. For a $N_{\text {var }}$ dimensional optimization problem, the habitat is an array with $1 \times N_{\mathrm{var}}$, which represents the current living position of the cuckoo. The habitat array is defined as follows [36, 40]:

$$
\text { habitat }=\left[X_{1}, X_{2}, \ldots, X_{N_{\text {var }}}\right] .
$$

A habitat's profit is obtained by evaluating the profit function $f_{p}$ for the habitat with $\left(X_{1}, X_{2}, \ldots, X_{N_{v a r}}\right)$; therefore, the following applies:

$$
\text { profit }=f_{p} \text { (habitat) }=f_{p}\left(X_{1}, X_{2}, \ldots, X_{N_{\text {var }}}\right) .
$$

For this relationship, CS maximizes the profit function. To use CS in cost-minimization problems, one can easily maximize the following profit function:

$$
\text { profit }=-\operatorname{cost}(\text { habitat })=-f_{c}\left(X_{1}, X_{2}, \ldots, X_{N_{\text {var }}}\right) .
$$

To begin the optimization algorithm, a candidate habitat matrix with the size $N_{\text {pop }} \times N_{\text {var }}$ is generated, and the initial cuckoo habitat is obtained. By nature, each cuckoo lays five to 20 eggs. These values are used as the upper and lower limits of eggs dedicated to each cuckoo at different iterations. Another habit of cuckoos is that they lay eggs within a maximum distance from their habitat, which is referred to as an egglaying radius (ELR) and is defined as follows:

$$
\begin{aligned}
\text { ELR }= & \beta \times \frac{\text { number of current cuckoo's eggs }}{\text { total number of eggs }} \\
& \times\left(\operatorname{var}_{\text {hi }}-\operatorname{var}_{\text {low }}\right)
\end{aligned}
$$

where $\operatorname{var}_{\text {hi }}$ and $\operatorname{var}_{\text {low }}$ are the upper and lower limits for the variables, respectively, and $\beta$ is an integer, supposed to handle the maximum value of ELR.

Each cuckoo begins to randomly lay eggs in another host birds' nest within her ELR. Figure 1(a) shows a clear perspective of a random egg-laying event in the ELR. The central red star is the initial habitat of the cuckoo with five eggs, and the small yellow stars are the eggs' new nest. Certain eggs that are more similar to the host birds' eggs can grow, hatch, be fed by the host birds, and become a mature cuckoo. Other eggs have no chance to grow, are detected by the host birds, and are destroyed. The habitat profit maximizes the number of surviving, hatched eggs. When young cuckoos grow up and become mature and as the time for egg-laying approaches, they immigrate to new and better habitats. The

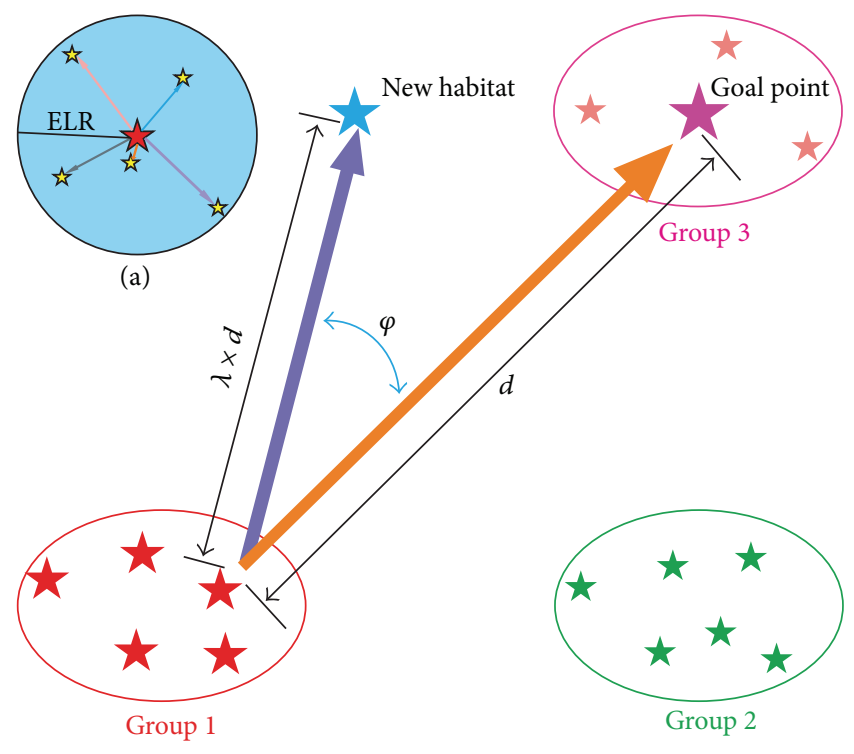

FIGURE 1: Random egg laying in an ELR and immigration of a sample cuckoo toward a goal habitat.

groups of cuckoos that form in different areas are recognizable using the K-means clustering method, and consequently the society with the best profit value is selected as the goal for immigration of other cuckoos.

Cuckoo movement towards a destination habitat is clearly shown in Figure 1. However, in this movement toward a goal point, each cuckoo only flies $\lambda \%$ of the total distance toward the goal habitat with the deviation $\varphi$ radians. $\lambda$ and $\varphi$ are random numbers and are defined as follows [36]:

$$
\begin{gathered}
\lambda \sim U(0,1), \\
\varphi \sim U(-\omega, \omega),
\end{gathered}
$$

where $\lambda \sim U(0,1)$ indicates that $\lambda$ is a random number uniformly distributed between 0 and $1 . \omega$ is a parameter that constrains the deviation from the goal habitat, and approximately $\pi / 6$ (radians) is recommended for $\omega$ for good convergence of the cuckoo population to a global maximum profit.

\section{The Available Data and Preprocessing}

4.1. The Available Data. Electricity consumption data used in this paper are collected every $30 \mathrm{~min}$ from the Australian Energy Market Operator (AEMO), New South Wales (NSW), Australia [41]. NSW with the largest population makes it Australia's most populous state; thus, accurate electricity consumption forecasting is crucial for planning and operating a power system of the city's sustainable development. The studied time range covers January 1st, 2013, to June 30th, 2013. The data are sampled with a certain time interval of $30 \mathrm{~min}$, so there are 48 data for one day. Figure 2 shows the variation trend of electricity consumption throughout the studied time range.

4.2. Abnormality Analysis and Data Preprocessing. When power systems are actually operated, any failures in 

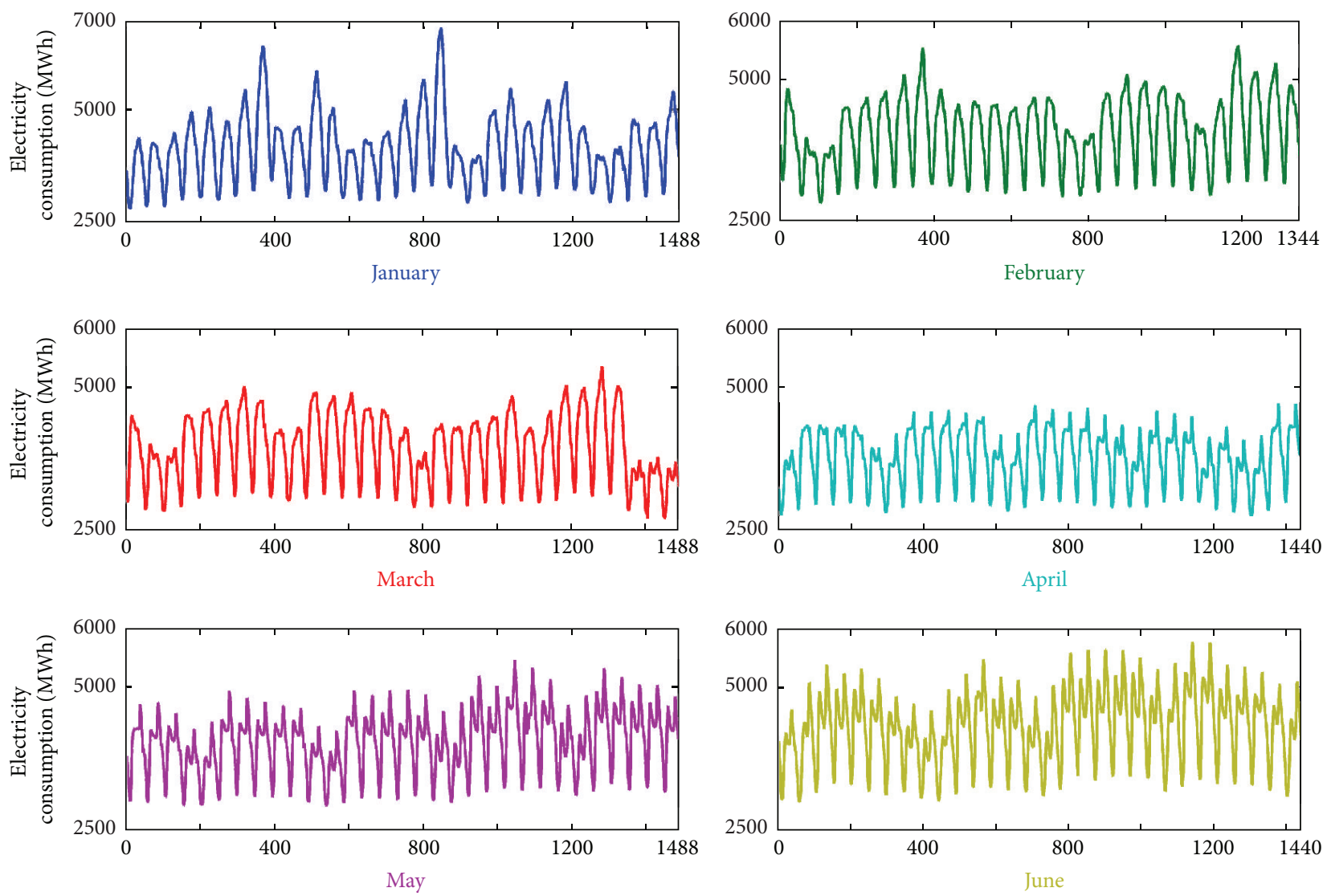

FIGURE 2: Electricity consumption data collected every $30 \mathrm{~min}$ in NSW from January 1st, 2013, to June 30th, 2013.

measurement, recording, conversion, and transmission losses may introduce an anomalous trend in the observed data, which is inconsistent with most observations. On the other hand, when the data acquisition system is normal, special events (such as load-shedding blackouts, power line maintenance, high-energy users, and large events) may produce abnormal changes in electricity consumption, which result in abnormal observations.

Suppose that the daily electricity consumption data includes 48 sample points. The abnormality analysis and its preprocessing are shown as follows.

Step 1. Abnormality analysis (discerning abnormal data): data will be considered abnormal if the difference between the data and adjacent data satisfies the following:

$$
|x(i)-x(i-1)|<\delta \quad(i=2,3, \ldots, 48),
$$

where $\delta$ is a constant.

Step 2. Compute the length of consecutive abnormal data occurrences, which is denoted by $l$.

Step 3. Abnormal data preprocessing: a day with abnormal data is the $n$th day, where $n$ is a constant. The correction values for electricity consumption are based on normal consumption data for the consecutive $n-1$ days before the abnormal day and the $k$ normal consumption data points on the $n$th day. Next, the correction values are defined as follows [13]:

$$
\begin{gathered}
D_{\text {mean }}(t)=\frac{1}{n-1} \sum_{i=1}^{n-1} D_{r}(i, t), \quad(t=1,2, \ldots, 48), \\
\bar{D}_{k}=\frac{1}{k} \sum_{i=1}^{k} D_{r}(n, t), \quad(t=1,2, \ldots, k), \\
\bar{D}_{\text {mean } k}=\frac{1}{k} \sum_{i=1}^{k} D_{\text {mean }}(t), \quad(t=1,2, \ldots, k), \\
D_{r}(n, t)=D_{\text {mean }}(t)-\left(\bar{D}_{\text {mean } k}-\bar{D}_{k}\right), \\
(t=k+1, \ldots, 48),
\end{gathered}
$$

where $D_{r}(i, t)$ is the consumption data at the time-point $t$ on the $i$ th day among $n-1$ days. $D_{\text {mean }}(t)$ is the mean of all consumption data at the time-point $t$ among $n-1$ days; $\bar{D}_{k}$ is the mean of the $k$ points for the normal consumption data on the $n$th day; $\bar{D}_{\text {mean } k}$ is the mean for all of the $k$ time-points among the $n-1$ days; and $D_{r}(n, t)$ is the consumption value at the time-point $t$ on the $n$th day.

We must select suitable values for $\delta$ and $l$ and then preprocess the abnormal data. To select the best values, $\delta$ has 
three values, $25 \mathrm{MWh}, 50 \mathrm{MWh}$, and $75 \mathrm{MWh}$, and $l$ has two values, 4 and 6 .

4.3. Feasibility Test. The grey model is superior to traditional forecasting approaches because it requires little sample data, easy calculation, and relatively high accuracy for shortterm forecasting. However, Wan et al. [42] noted that a slowly increasing data sequence is suitable for establishing a $\mathrm{GM}(1,1)$, but a rapidly increasing data sequence is unsuitable for constructing a $\mathrm{GM}(1,1)$. Therefore, the class ratio of the original data is calculated to determine whether it is suitable for directly constructing a grey model.

The class ratio $\sigma^{0}(k)$ is defined as follows:

$$
\sigma^{0}(k)=\frac{x^{(0)}(k-1)}{x^{(0)}(k)}, \quad k=2,3, \ldots m .
$$

If the values for $\sigma^{0}(k)(k=2,3, \ldots, m)$ are in the range $e^{-2 /(m+1)}$ to $e^{2 /(m+1)}, x^{(0)}$ is suitable for modeling a $\operatorname{GM}(1,1)$. If the values for $\sigma^{0}(k)(k=2,3, \ldots, m)$ are out of the range, $x^{(0)}$ must be log-transformed to force the class ratio into the range. This procedure is the feasibility test.

\section{A Case Study}

5.1. Simulation Procedure. Optimized GM(1,1)s are constructed to select the best forecasting strategy based on the data before the predicted day; the best forecasting strategy is then used to predict the forecasted day. The simulation procedure for forecasting electricity consumption based on the optimized GM(1,1) is described as follows.

Process 1 . Let the current forecasted day (CFD) be the day before the forecasted day. Select the data for modeling the $\mathrm{GM}(1,1)$ from a short-term perspective in days and a longterm perspective in weeks:

$$
\begin{aligned}
& D_{1 t}^{(0)}=\left\{D_{1 t}^{(0)}(k) \mid k=1,2, \ldots, \lambda\right\}, \\
& D_{2 t}^{(0)}=\left\{D_{2 t}^{(0)}(k) \mid k=1,2, \ldots, \lambda\right\},
\end{aligned}
$$

where $D_{1 t}^{(0)}$ is the data used to construct the grey model at time $t$ from the $\lambda$ five days before the CFD, which reflects the short-term characteristics and is referred to as Scheme 1. $D_{1 t}^{(0)}$ is the data used to construct the grey model at time $t$ on the same day for the previous $\lambda$ weeks, which reflects the longterm characteristics and is referred to as Scheme 2; $\lambda$ is the number of days used to establish the $\operatorname{GM}(1,1)$, and $\lambda$ is fixed at 5. $t$ represents the time- points for each half-hour over a day, and $t=1,2, \ldots, 48$.

Process 2. Apply the abnormality analysis and preprocess the abnormal data in accordance with Section 3.2. $\delta$ and $l$ are alterable; notably, the combination $(\delta, l)$ includes six cases: (25 MWh, 4), (25 MWh, 6), (50 MWh, 4), (50 MWh, 6), (75 MWh, 4), and (75 MWh, 6). Thereafter, the preprocessing data are transformed to better suit a grey model in accordance with Section 3.3. The new obtained electricity consumption series are denoted by $d_{1 t}^{(0)}$ for the short-term data and $d_{2 t}^{(0)}$ for the long-term data.

Process 3. Construct the $\mathrm{GM}(1,1)$ s based on the sample data $d_{1 t}^{(0)}$ and $d_{2 t}^{(0)}$. The parameter $\alpha$ in (4) is optimized using the iterative and cuckoo search algorithms described in Section 2, respectively. Intuitively, the CS algorithm is more reasonable than the iterative optimization algorithm because historical data are used to construct the grey model, which yield a better $\alpha$ value. For the above two schemes, the corresponding forecasted consumption at time $t$ on the CFD is obtained, respectively, as follows for $\widehat{d}_{1 t}^{(0)}(\lambda+1)$ and $\widehat{d}_{2 t}^{(0)}(\lambda+$ 1):

$$
\begin{array}{ll}
\widehat{d}_{1 t}^{(0)}(\lambda+1)=\widehat{d}_{1 t}^{(1)}(\lambda+1)-\widehat{d}_{1 t}^{(1)}(\lambda), & (t=1,2, \ldots, 48), \\
\widehat{d}_{2 t}^{(0)}(\lambda+1)=\widehat{d}_{2 t}^{(1)}(\lambda+1)-\widehat{d}_{2 t}^{(1)}(\lambda), & (t=1,2, \ldots, 48) .
\end{array}
$$

Process 4 . We average the same days from the last five weeks before the CFD at time $t$, which is denoted by $\bar{D}_{5 \text {-weeks }}(t)(t=$ $1,2, \ldots, 48)$. We divide the CFD into four parts as peaks and valleys of the electricity consumption data to effectively relieve the load change intensity for each segment and to improve the $\operatorname{GM}(1,1)$ forecasting accuracy [13]. Upon implementation of this method, the following simple and effective partition method is proposed.

Part 1. The midnight part is from 0:00 to the time of the first peak.

Part 2. The morning part is from the time of the first peak to the time of the first valley.

Part 3. The afternoon part is from the time of the first valley to the time of the evening peak.

Part 4. The evening part is from the time of the evening peak to 24:00.

The grey correlation coefficients of for each part between the two forecasting electricity consumption data and the CFD consumption are calculated using (23), respectively. The grey correlation coefficient of different schemes usually varies in different parts. The scheme with the greatest grey correlation coefficient for each part is used as the forecasting scheme; the CFD forecasting values are obtained by linking each part of the adopted forecasting values:

$$
\begin{gathered}
\varepsilon(k)=\frac{\min _{k} \Delta(k)+\rho \cdot \max _{k} \Delta(k)}{\Delta(k)+\rho \cdot \max _{k} \Delta(k)}, \\
r=\frac{1}{m} \sum_{k=1}^{m} \varepsilon(k),
\end{gathered}
$$

where $\varepsilon(k)$ is the correlation coefficient at each point, $\rho$ is typically 0.5 , and $r$ is the grey correlation coefficient.

Process 5. After the above four processes are completed, we determine the best forecasting scheme that corresponds to 


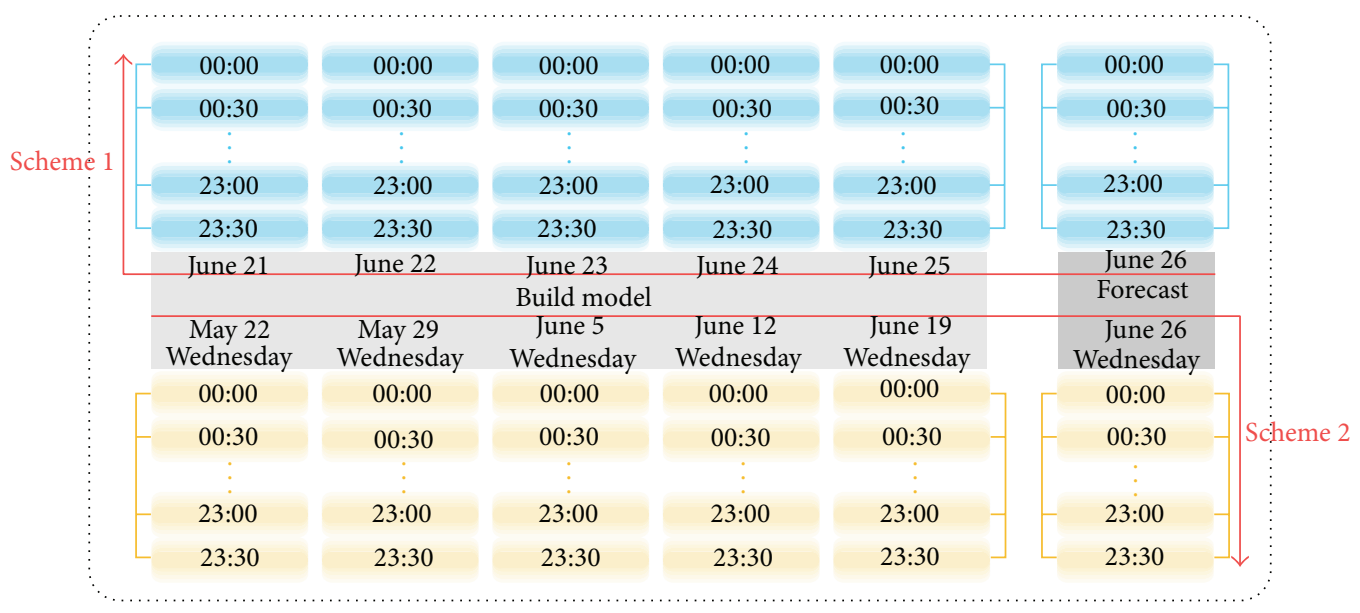

Figure 3: The data format for Scheme 1 and Scheme 2.

each forecasting part. Next, the CFD is shifted to the actual forecasted day, and the simulation procedure is reapplied. Therefore, the optimized $\operatorname{GM}(1,1)$ is used to forecast the consumption series for the actual forecasted day process by process.

Process 6. The optimized GM(1,1) models (IAGM and CSGM) are compared with an autoregressive integrated moving average model (ARIMA) and GM(1,1) (GM) when the NSW electricity consumption is forecasted.

\subsection{Forecasting Electricity Consumption in the NSW}

5.2.1. Analysis of Forecasting Results. We forecasted the electricity consumption data for June 26th, 2013, using the GM, IAGM, CSGM, and ARIMA models. The corresponding data format is defined in Figure 3. The above six processes were executed, and the forecasting results are shown in Figure 4.

(1) At the top of Figure 4, the white words on the red background show six cases (situations) in the abnormality data analysis. Delta $\delta$ has three values, and $l$ has two values; thus, a combination of six cases will yield six different preprocessing results.

(2) From the short-term (Scheme 1) and long-term (Scheme 2) perspectives, we selected two different data sets for modeling and then performed the abnormality analysis and feasibility test.

(3) The electricity consumption was forecasted using the IAGM model; then, the grey correlation coefficients were calculated for each part in Schemes 1 and 2. The best scheme for each part was selected using the grey correlation coefficient for the corresponding part. The best schemes for the four parts in Case 1 are as follows. The best scheme for Part 1 is Scheme 2; the best scheme for the other three parts is Scheme 1. For the remaining five cases, the rounded rectangle in orange and green represents the best forecasting schemes for each part. For example, in Case 4, Part 1 and Part 4 are orange and Part 2 and Part 3 are green; thus, the best schemes in the order of the parts are Scheme 2, Scheme 1, Scheme 1, and Scheme 2.

(4) Thereafter, the CSGM model under the best scheme obtained using IAGM is applied to forecast the electricity consumption. The forecasting results are shown in Figures 4(a), 4(b), and 4(c).

(5) Figure 4(a) shows the average error for six different cases using two different forecasting methods. For Case 1, the average error values for IAGM and CSGM are $4.1137 \%$ and $5.7342 \%$, respectively, which is unsatisfactory for electricity consumption forecasting and management. Case 2 is also unsatisfactory, for which the IAGM and CSGM mean error values are $7.3053 \%$ and $4.8228 \%$, respectively. For Case 4 , the CSGM error meets the power market requirements; however, the $4.7591 \%$ IAGM error is not satisfactory. In addition, the other cases (Case 3, Case 5, and Case 6) yielded smaller errors and more satisfactory outcomes.

(6) The forecasting errors at each IAGM and ISGM timepoint are presented in Figures 4(b) and 4(c). For the CSGM Cases 3-6 and the IAGM Cases 3, 5, and 6 , the error curves show small fluctuations. Case 3 , Case 5, and Case 6 were used for the forecasting results. For Part 1 (the midnight part), the forecasting errors for the six cases using the two methods are the same because the electricity consumption for the midnight part is stable and only slightly changes. The differences between IAGM Parts 3-4 and CSGM are slight, whereas the forecasting errors for the CSGM Part 2 (the morning part) were significantly better than for the IAGM. In this study, electricity consumption decreases with the greatest fluctuation in the morning part from 9:00 to 15:30. The above analysis indicates that the CSGM better manages large data fluctuations than the IAGM.

(7) The best forecasting results were obtained for the CSGM model in Case 6, for which the average error is $2.0667 \%$. 


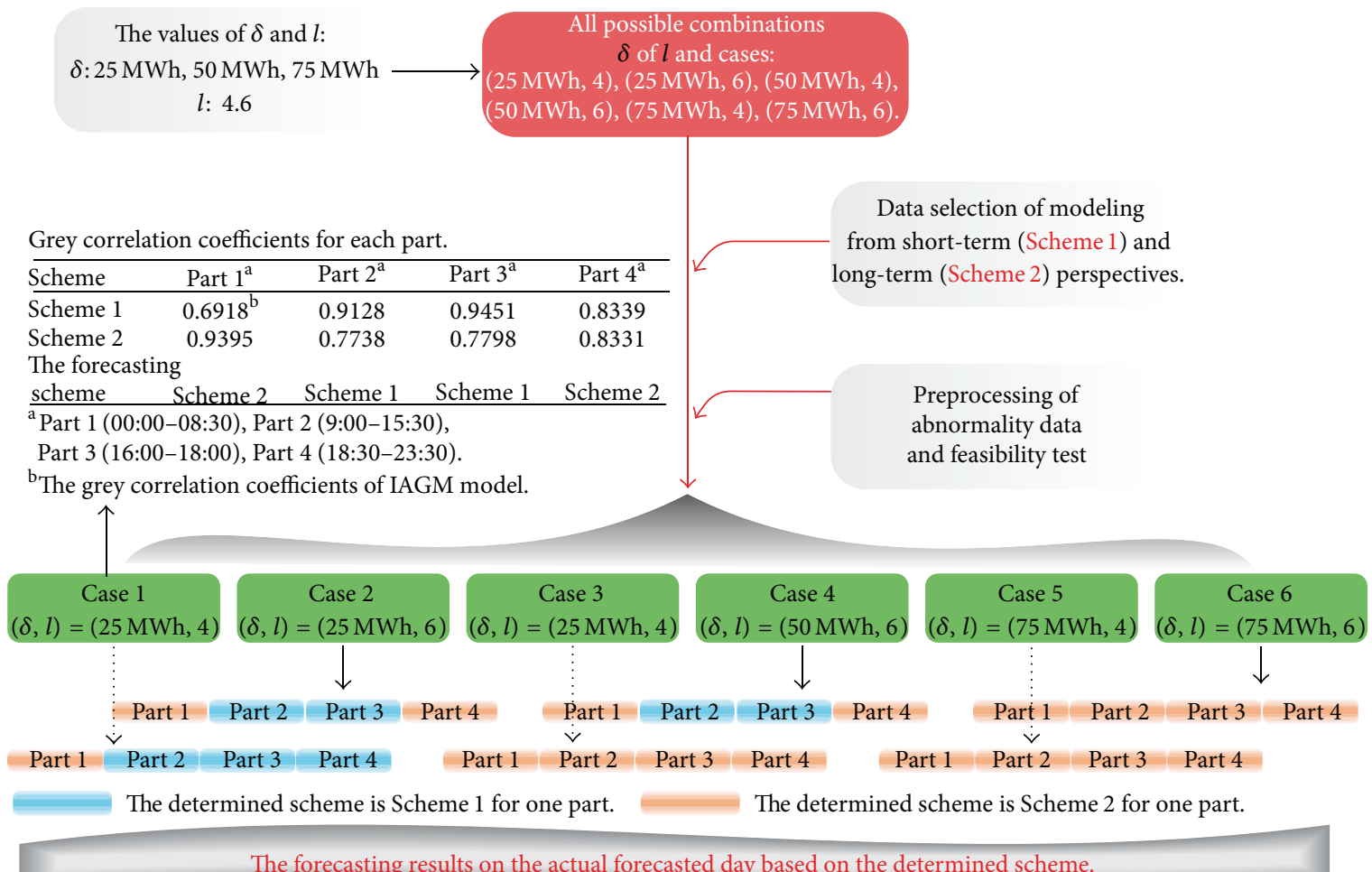

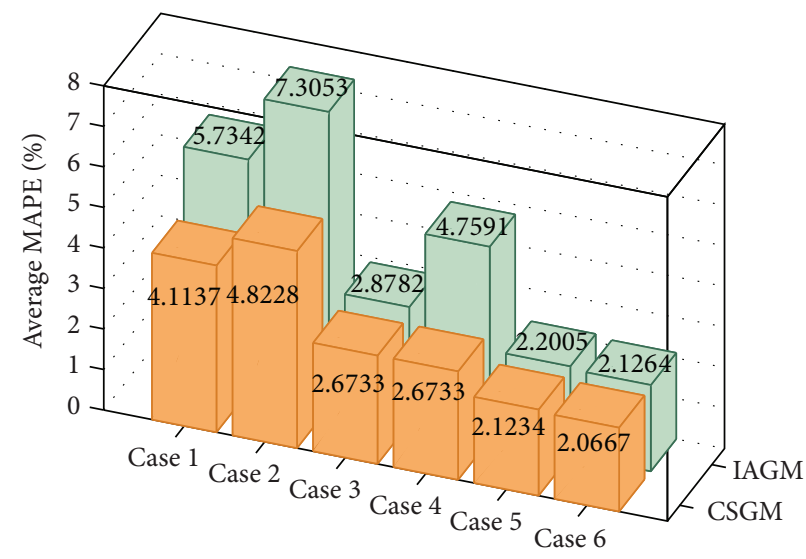

(a)

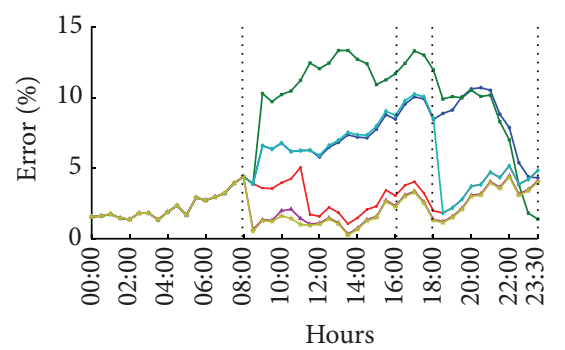

(b)

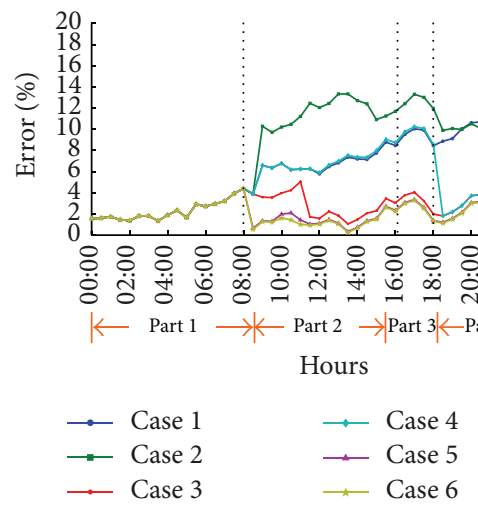

(c)

FIGURE 4: The forecasting results for the IAGM and CSGM models based on six different cases. (a) The average errors of IGAM and CSGM and the error values. (b) The forecasting errors of CSGM in six cases. The black dashed lines represent fences of different part. (c) The forecasting errors of IAGM in six cases. 


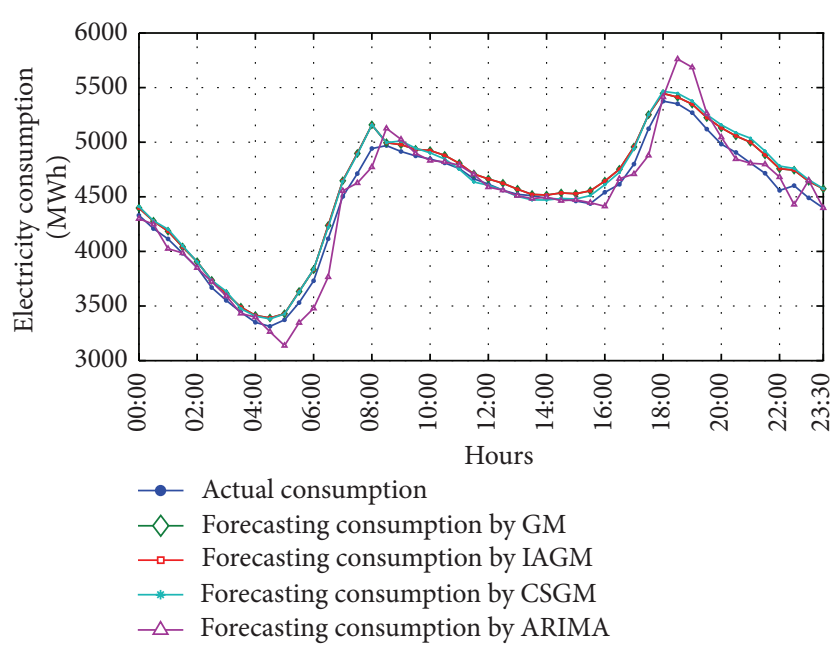

FIgURE 5: The forecasting results and actual values.

Figure 5 and Table 1 show the forecasting results for the ARIMA, GM, IAGM (Case 6), and CSGM (Case 6), respectively. Figure 5 and Table 1 indicate the following.

(1) The four forecasting methods, except the ARIMA model, yield good fitting results for the original electricity consumption data.

(2) The GM forecasting results are similar to IAGM. The forecasting curves show that the forecasting values for the GM almost coincide with the IAGM for all four parts.

(3) For Parts 1-3, GM, IAGM, and CSGM yield satisfactory forecasting results. However, for Part 4, all three models yielded relatively large errors, perhaps because the electricity consumption fluctuation in the evening is greater than at midnight as well as during the morning and afternoon.

(4) A highly inaccurate estimate was observed at or near the yielding point of the original data in all four models.

(5) The average forecasting errors for GM, IAGM, CSGM, and ARIMA are $2.12 \%, 2.13 \%, 2.07 \%$, and $2.04 \%$, respectively, which may meet the electricity prediction and management requirements.

(6) The maximum forecasting errors for GM, IAGM, and CSGM are similar; specifically, they are $4.39 \%, 4.39 \%$, and $4.89 \%$, respectively. The maximum forecasting error for the ARIMA model is $8.49 \%$, which is significantly larger than that for the other three models.

(7) The CSGM performed better than the GM and IAGM. Moreover, the IAGM performed similar to the GM. Although the average ARIMA error is the lowest among the forecasting models, the maximum ARIMA error is markedly higher than the other three models and reaches $8.49 \%$. Thus, more analyses are necessary to determine whether ARIMA is a suitable electricity forecasting approach.

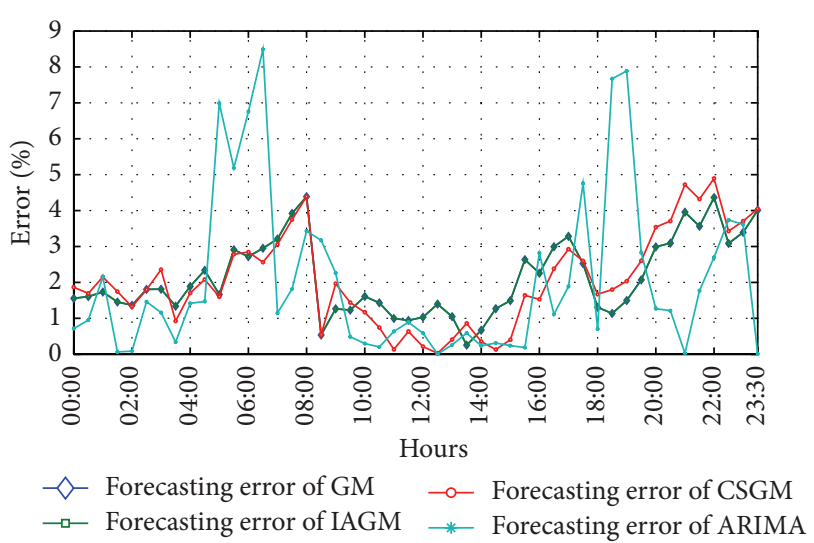

FIgURE 6: The GM, IAGM, CSGM, and ARIMA forecasting errors.

5.2.2. Forecasting Error Analysis Using Statistical Theory. Figure 6 shows the forecasting errors for the four models, and the ARIMA error fluctuates greatly. The frequency diagram and box plot for the forecasting errors are shown in Figure 7. Figure 7 (a) shows that the errors are mostly in the interval 0 to $3.40 \%$. A few errors are greater than $3.40 \%$ and less than $5.1 \%$, and the maximum and second largest error intervals only include the ARIMA forecasting error. These data demonstrate that ARIMA is unsuitable for forecasting electricity consumption in the NSW. As shown in Figure 7(b), in addition to the ARIMA model, the other three quartiles (the lower, median, and upper quartiles) calculated for the other three models have similar variations in the range length. The whiskers in the box plot indicate the primary range for the data, in which the lowest data are 1.5 times the interquartile range of the lower quartile and the highest data are 1.5 times the interquartile range of the upper quartile (see Figure $7(\mathrm{c})$ ). The outliers, which are not included between the whiskers, are represented by red small circles. The GM, IAGM, and CSGM forecasting errors do not have outliers, while the number of outliers for the ARIMA reaches 5 .

On the one hand, due to the lack of large-scale storage in the electric industry, supply is adjusted to match consumption in real time. High forecasting errors will produce an imbalance between electricity supply and consumption. Underestimating electricity consumption will lead to an electricity shortage, and an overestimate would waste precious energy resources [43]. In addition, the normal power grid operation should increase the capacity reserve, which is an additional supply to account for transmission losses. Grid operators have the capacity in reserve to respond to electricity high consumption periods and unplanned power plant outages. A high forecasting error will lead to inaccurate capacity reserve estimates and then to an administrative risk for the power grid and increased operation costs. Bunn and Farmer noted that a $1 \%$ increase in a forecasting error may lead to a $£ 10$ million increase in the operating costs [44]. Therefore, it is significantly important to forecast electricity demand accurately. Accurate electricity consumption forecasts can aid power generators in scheduling their power station operations to match the installed capacity. Small and stable errors 
TABLE 1: Electricity consumption forecasting results.

\begin{tabular}{|c|c|c|c|c|c|c|c|c|c|}
\hline \multirow{2}{*}{$\begin{array}{l}\text { Time- } \\
\text { point }\end{array}$} & \multirow[b]{2}{*}{$\begin{array}{l}\text { Actual value } \\
\text { (MWh) }\end{array}$} & \multicolumn{2}{|l|}{ GM } & \multicolumn{2}{|c|}{ IAGM } & \multicolumn{2}{|c|}{ CSGM } & \multicolumn{2}{|c|}{ ARIMA $(2,2,1)$} \\
\hline & & $\begin{array}{c}\text { Forecasting } \\
\text { values }\end{array}$ & $\begin{array}{c}\text { Error }^{\mathrm{a}} \\
(\%)\end{array}$ & $\begin{array}{c}\text { Forecasting } \\
\text { values }\end{array}$ & $\begin{array}{c}\text { Error } \\
(\%)\end{array}$ & $\begin{array}{c}\text { Forecasting } \\
\text { values }\end{array}$ & $\begin{array}{c}\text { Error } \\
(\%)\end{array}$ & $\begin{array}{c}\text { Forecasting } \\
\text { values }\end{array}$ & $\begin{array}{c}\text { Error } \\
(\%)\end{array}$ \\
\hline $0: 00$ & 4330.270 & 4397.628 & 1.56 & 4397.713 & 1.56 & 4411.040 & 1.87 & 4299.163 & 0.72 \\
\hline $0: 30$ & 4209.265 & 4276.840 & 1.61 & 4276.919 & 1.61 & 4280.632 & 1.70 & 4249.257 & 0.95 \\
\hline $1: 00$ & 4114.000 & 4185.289 & 1.73 & 4185.387 & 1.74 & 4202.551 & 2.15 & 4025.156 & 2.16 \\
\hline $1: 30$ & 3982.655 & 4040.614 & 1.46 & 4040.685 & 1.46 & 4052.158 & 1.75 & 3985.292 & 0.07 \\
\hline $2: 00$ & 3853.080 & 3905.699 & 1.37 & 3905.752 & 1.37 & 3904.051 & 1.32 & 3849.782 & 0.09 \\
\hline $2: 30$ & 3668.835 & 3735.070 & 1.81 & 3735.142 & 1.81 & 3734.825 & 1.80 & 3722.507 & 1.46 \\
\hline 3:00 & 3550.665 & 3614.975 & 1.81 & 3615.062 & 1.81 & 3634.288 & 2.36 & 3591.899 & 1.16 \\
\hline $3: 30$ & 3443.545 & 3489.662 & 1.34 & 3489.750 & 1.34 & 3475.360 & 0.92 & 3431.846 & 0.34 \\
\hline $4: 00$ & 3350.500 & 3413.786 & 1.89 & 3413.850 & 1.89 & 3407.637 & 1.71 & 3397.992 & 1.42 \\
\hline $4: 30$ & 3313.335 & 3390.756 & 2.34 & 3390.803 & 2.34 & 3382.276 & 2.08 & 3264.648 & 1.47 \\
\hline $5: 00$ & 3373.360 & 3429.484 & 1.66 & 3429.524 & 1.66 & 3427.320 & 1.60 & 3137.584 & $6.99^{b}$ \\
\hline $5: 30$ & 3530.420 & 3633.135 & 2.91 & 3633.220 & 2.91 & 3628.695 & 2.78 & 3347.417 & $5.18^{\mathrm{b}}$ \\
\hline $6: 00$ & 3730.750 & 3832.116 & 2.72 & 3832.177 & 2.72 & 3836.934 & 2.85 & 3478.720 & $6.76^{b}$ \\
\hline $6: 30$ & 4115.280 & 4236.704 & 2.95 & 4236.766 & 2.95 & 4220.757 & 2.56 & 3765.914 & $8.49^{b}$ \\
\hline 7:00 & 4502.655 & 4647.155 & 3.21 & 4647.238 & 3.21 & 4640.427 & 3.06 & 4554.320 & 1.15 \\
\hline 7:30 & 4712.280 & 4897.057 & 3.92 & 4897.194 & 3.92 & 4888.935 & 3.75 & 4626.589 & 1.82 \\
\hline 8:00 & 4941.845 & 5158.762 & 4.39 & 5158.978 & 4.39 & 5158.713 & 4.39 & 4772.266 & 3.43 \\
\hline $8: 30$ & 4969.065 & 4995.615 & 0.53 & 4995.667 & 0.54 & 4995.996 & 0.54 & 5126.765 & 3.17 \\
\hline 9:00 & 4915.090 & 4977.279 & 1.27 & 4977.374 & 1.27 & 5011.611 & 1.96 & 5026.143 & 2.26 \\
\hline $9: 30$ & 4874.630 & 4934.493 & 1.23 & 4934.557 & 1.23 & 4944.742 & 1.44 & 4898.405 & 0.49 \\
\hline 10:00 & 4845.960 & 4923.899 & 1.61 & 4923.984 & 1.61 & 4902.575 & 1.17 & 4831.506 & 0.30 \\
\hline $10: 30$ & 4810.585 & 4879.438 & 1.43 & 4879.522 & 1.43 & 4846.219 & 0.74 & 4820.355 & 0.20 \\
\hline 11:00 & 4758.920 & 4806.381 & 1.00 & 4806.484 & 1.00 & 4752.485 & 0.14 & 4789.164 & 0.64 \\
\hline 11:30 & 4665.005 & 4708.921 & 0.94 & 4709.015 & 0.94 & 4635.415 & 0.63 & 4706.608 & 0.89 \\
\hline $12: 00$ & 4615.740 & 4663.379 & 1.03 & 4663.437 & 1.03 & 4605.949 & 0.21 & 4588.729 & 0.59 \\
\hline $12: 30$ & 4560.465 & 4624.130 & 1.40 & 4624.185 & 1.40 & 4559.143 & 0.03 & 4560.645 & 0.00 \\
\hline 13:00 & 4522.080 & 4569.492 & 1.05 & 4569.526 & 1.05 & 4503.554 & 0.41 & 4510.220 & 0.26 \\
\hline 13:30 & 4510.240 & 4521.704 & 0.25 & 4521.722 & 0.25 & 4471.516 & 0.86 & 4483.658 & 0.59 \\
\hline 14:00 & 4486.135 & 4516.147 & 0.67 & 4516.169 & 0.67 & 4470.371 & 0.35 & 4496.701 & 0.24 \\
\hline 14:30 & 4478.710 & 4535.532 & 1.27 & 4535.573 & 1.27 & 4484.597 & 0.13 & 4464.722 & 0.31 \\
\hline 15:00 & 4461.810 & 4528.393 & 1.49 & 4528.434 & 1.49 & 4479.814 & 0.40 & 4472.497 & 0.24 \\
\hline 15:30 & 4437.840 & 4554.719 & 2.63 & 4554.768 & 2.63 & 4510.697 & 1.64 & 4446.137 & 0.19 \\
\hline $16: 00$ & 4541.070 & 4643.533 & 2.26 & 4643.599 & 2.26 & 4610.473 & 1.53 & 4413.194 & 2.82 \\
\hline $16: 30$ & 4614.100 & 4752.256 & 2.99 & 4752.335 & 3.00 & 4723.989 & 2.38 & 4665.412 & 1.11 \\
\hline 17:00 & 4799.055 & 4956.608 & 3.28 & 4956.701 & 3.28 & 4939.399 & 2.92 & 4708.471 & 1.89 \\
\hline $17: 30$ & 5122.475 & 5251.827 & 2.53 & 5251.924 & 2.53 & 5255.480 & 2.60 & 4878.819 & 4.76 \\
\hline 18:00 & 5375.885 & 5446.141 & 1.31 & 5446.216 & 1.31 & 5465.649 & 1.67 & 5413.701 & 0.70 \\
\hline 18:30 & 5350.900 & 5411.714 & 1.14 & 5411.789 & 1.14 & 5447.303 & 1.80 & 5761.367 & $7.67^{\mathrm{b}}$ \\
\hline 19:00 & 5269.615 & 5348.454 & 1.50 & 5348.550 & 1.50 & 5376.871 & 2.04 & 5685.330 & $7.89^{b}$ \\
\hline $19: 30$ & 5119.510 & 5225.517 & 2.07 & 5225.610 & 2.07 & 5252.610 & 2.60 & 5264.521 & 2.83 \\
\hline $20: 00$ & 4982.405 & 5131.309 & 2.99 & 5131.410 & 2.99 & 5158.740 & 3.54 & 5045.731 & 1.27 \\
\hline $20: 30$ & 4905.600 & 5057.356 & 3.09 & 5057.450 & 3.10 & 5087.582 & 3.71 & 4846.094 & 1.21 \\
\hline 21:00 & 4809.135 & 4999.369 & 3.96 & 4999.489 & 3.96 & 5036.191 & 4.72 & 4807.723 & 0.03 \\
\hline 21:30 & 4715.035 & 4883.033 & 3.56 & 4883.162 & 3.57 & 4918.670 & 4.32 & 4798.520 & 1.77 \\
\hline $22: 00$ & 4558.515 & 4757.412 & 4.36 & 4757.540 & 4.37 & 4781.606 & 4.89 & 4681.545 & 2.70 \\
\hline $22: 30$ & 4602.065 & 4744.022 & 3.08 & 4744.124 & 3.09 & 4759.853 & 3.43 & 4430.017 & 3.74 \\
\hline 23:00 & 4489.415 & 4641.814 & 3.39 & 4641.920 & 3.40 & 4655.801 & 3.71 & 4651.653 & 3.61 \\
\hline $23: 30$ & 4397.565 & 4573.946 & 4.01 & 4574.034 & 4.01 & 4575.651 & 4.05 & 4398.089 & 0.01 \\
\hline & mum forecast & rror (\%) & 4.39 & & 4.39 & & 4.89 & & 8.49 \\
\hline & rage forecastin & ror $(\%)$ & 2.12 & & 2.13 & & 2.07 & & 2.04 \\
\hline
\end{tabular}

${ }^{a}$ The error is defined as follows: error $=\mid$ Forecasting value - Actual value $\mid /$ actual value $* 100 \%$.

${ }^{\mathrm{b}}$ The forecasting error value is greater than $5 \%$. The specific time-points are 5:00, 5:30, 6:00, 6:30, 18:30, and 19:00. 


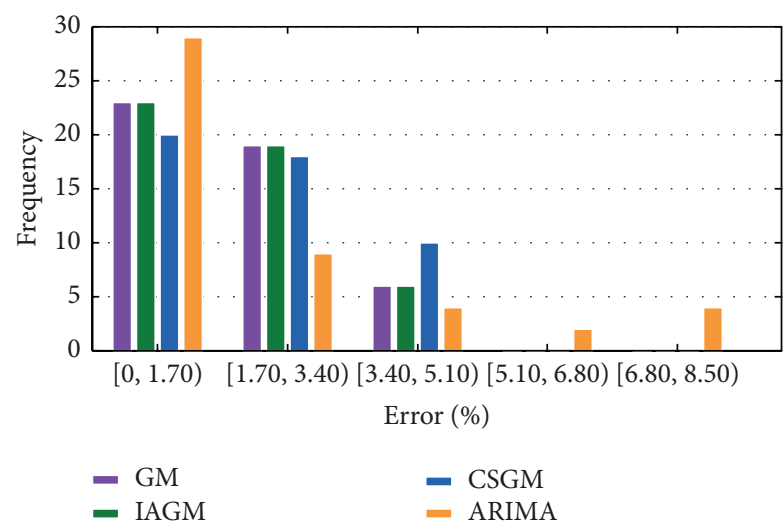

(a)

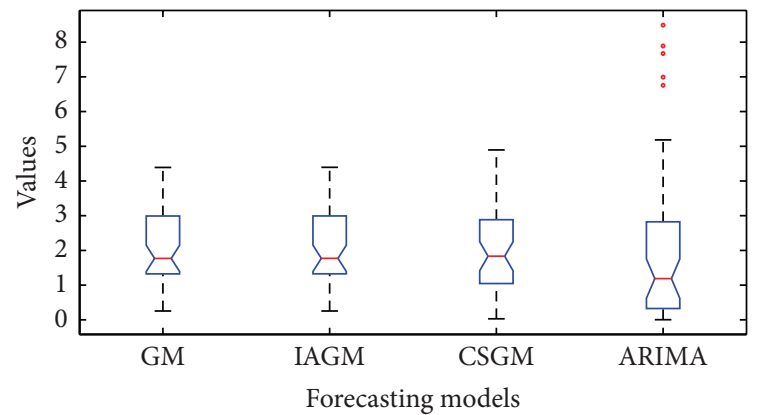

(b)

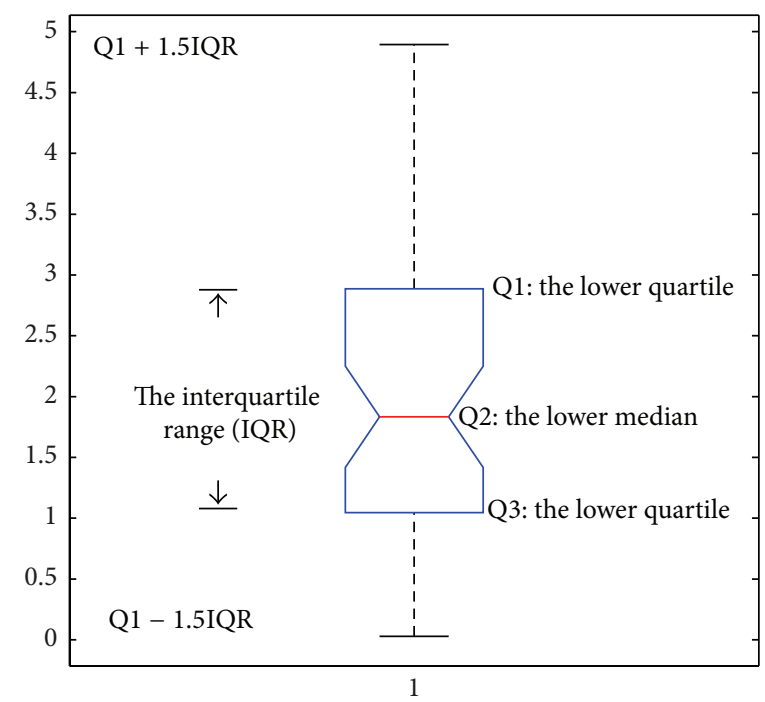

(c)

FIGURE 7: The frequency diagram and box plot for the GM, IAGM, CSGM, and ARIMA models. (a) The frequency diagram of four forecasting models. The interval on the $x$-axis is the range of error. (b) The box plot of four forecasting models. Box plots are a way of graphically depicting groups of numerical data through their quartiles. Whiskers from the box indicate variability outside the upper and lower quartiles. Outliers are plotted in red circles. (c) A detailed graphic presentation of a box plot. The central mark is the median, the edges of the box are the lower and upper quartiles, the whiskers extend to the most extreme data points not considering outliers, and outliers are plotted individually.

in forecasting approaches are certainly necessary. Although the average ARIMA error is the lowest among the four forecasting methods, ARIMA is unsuitable for forecasting electricity consumption in this case. The CSGM forecasting performance is superior to the other models, and the GM forecasting performance is similar to IAGM.

\section{Conclusion}

The one-day-ahead electricity consumption forecast is an extremely important problem in electricity load planning, secure operation, and energy expenditure/cost economy. However, electricity consumption data are affected by multiple uncertain factors, such as climate change and the social environment. Grey theory can be used to construct a forecasting model using uncertain and insufficient information, and it meets the requirements of electricity consumption prediction.
This paper proposes a grey-theory-based model, CSGM, to forecast electricity consumption. First, to reflect the similarity in electricity data for different days or weeks at the same time-point, the original series are selected from short- and long-term perspectives. Excellent GM(1,1) performance requires a slowly increasing data series; thus, data preprocessing includes abnormality and feasibility tests to improve the forecasting performance. To further enhance electricity consumption forecasting precision, two optimized algorithms, IA and CS, are used to select suitable parameters for $\operatorname{GM}(1,1)$. Finally, when the data varies smoothly, the GM $(1,1)$ results will be more accurate. The forecasted day is divided into four smooth parts based on the grey correlation coefficient for each part, and the best forecasting scheme is determined. In addition, to evaluate the applicability of the one-day-ahead forecast in a New South Wales power grid of Australia, the CSGM was compared with the GM, IAGM, and ARIMA models. According to the electricity consumption 
forecasting analysis and errors, CSGM outperforms the other models; the forecasting performance of GM and IAGM meets the electricity market requirement. However, ARIMA is not suitable to forecast electricity consumption in this study because the forecasting error is fluctuated dramatically.

\section{Abbreviations}

IA: Iterative algorithm

CS: Cuckoo search algorithm

GM: $\quad$ Grey model

ARIMA: Autoregressive integrated moving average model

EMD: Empirical model decomposition

NSW: New South Wales

AGO: Accumulating generation operator

IAGO: Inverse accumulated generating operation

IAGM: $\quad \mathrm{GM}(1,1)$ optimized using the IA

CSGM: $\quad$ GM(1,1) optimized using CS

ELR: $\quad$ Egg-laying radius

AEMO: Australian Energy Market Operator

CFD: Current forecasted day

Scheme 1: Short-term selection

Scheme 2: Long-term selection.

\section{Conflict of Interests}

The authors declare that there is no conflict of interests regarding the publication of this paper.

\section{Acknowledgment}

This work was supported by the National Natural Science Foundation of China (Grant no. 70973015).

\section{References}

[1] J. Wang, X. Ma, J. Wu, and Y. Dong, "Optimization models based on GM $(1,1)$ and seasonal fluctuation for electricity demand forecasting," International Journal of Electrical Power \& Energy Systems, vol. 43, no. 1, pp. 109-117, 2012.

[2] U. Al-mulali, H. G. Fereidouni, and J. Y. M. Lee, "Electricity consumption from renewable and non-renewable sources and economic growth: evidence from Latin American countries," Renewable and Sustainable Energy Reviews, vol. 30, pp. 290-298, 2014.

[3] N. M. Odhiambo, "Electricity consumption and economic growth in South Africa: a trivariate causality test," Energy Economics, vol. 31, no. 5, pp. 635-640, 2009.

[4] S. Ghosh, "Electricity consumption and economic growth in India," Energy Policy, vol. 30, no. 2, pp. 125-129, 2002.

[5] M. Shahbaz and H. H. Lean, "The dynamics of electricity consumption and economic growth: a revisit study of their causality in Pakistan," Energy, vol. 39, no. 1, pp. 146-153, 2012.

[6] C. F. Tang and E. C. Tan, "Exploring the nexus of electricity consumption, economic growth, energy prices and technology innovation in Malaysia," Applied Energy, vol. 104, pp. 297-305, 2013.
[7] S.-H. Yoo and S.-Y. Kwak, "Electricity consumption and economic growth in seven South American countries," Energy Policy, vol. 38, no. 1, pp. 181-188, 2010.

[8] M. Thoma, "Electrical energy usage over the business cycle," Energy Economics, vol. 26, no. 3, pp. 463-485, 2004.

[9] S.-H. Yoo, "The causal relationship between electricity consumption and economic growth in the ASEAN countries," Energy Policy, vol. 34, no. 18, pp. 3573-3582, 2006.

[10] J. Yuan, C. Zhao, S. Yu, and Z. Hu, "Electricity consumption and economic growth in China: cointegration and co-feature analysis," Energy Economics, vol. 29, no. 6, pp. 1179-1191, 2007.

[11] Z. Zhao, J. Wang, J. Zhao, and Z. Su, "Using a Grey model optimized by Differential Evolution algorithm to forecast the per capita annual net income of rural households in China," Omega, vol. 40, no. 5, pp. 525-532, 2012.

[12] D.-C. Li, C.-J. Chang, C.-C. Chen, and W.-C. Chen, "Forecasting short-term electricity consumption using the adaptive Greybased approach: an Asian case," Omega, vol. 40, no. 6, pp. 767773, 2012.

[13] M. Jin, X. Zhou, Z. M. Zhang, and M. M. Tentzeris, "Shortterm power load forecasting using Grey correlation contest modeling," Expert Systems with Applications, vol. 39, no. 1, pp. 773-779, 2012.

[14] J. M. Abreu, F. Câmara Pereira, and P. Ferrão, “Using pattern recognition to identify habitual behavior in residential electricity consumption," Energy and Buildings, vol. 49, pp. 479-487, 2012.

[15] R. Gutiérrez, R. Gutiérrez-Sánchez, and A. Nafidi, “Electricity consumption in Morocco: stochastic Gompertz diffusion analysis with exogenous factors," Applied Energy, vol. 83, no. 10, pp. 1139-1151, 2006.

[16] J. Antoch, L. Prchal, M. R. de Rosa, and P. Sarda, "Electricity consumption prediction with functional linear regression using spline estimators," Journal of Applied Statistics, vol. 37, no. 12, pp. 2027-2041, 2010.

[17] Z. Mohamed and P. Bodger, "Forecasting electricity consumption in New Zealand using economic and demographic variables," Energy, vol. 30, no. 10, pp. 1833-1843, 2005.

[18] L. S. Aiken, S. G. West, and S. C. Pitts, "Multiple linear regression," in Handbook of Psychology, Wiley, Chichester, UK, 2003.

[19] Y. Dong, J. Wang, H. Jiang, and J. Wu, "Short-term electricity price forecast based on the improved hybrid model," Energy Conversion and Management, vol. 52, no. 8-9, pp. 2987-2995, 2011.

[20] Y. Ohtsuka, T. Oga, and K. Kakamu, "Forecasting electricity demand in Japan: a Bayesian spatial autoregressive ARMA approach," Computational Statistics \& Data Analysis, vol. 54, no. 11, pp. 2721-2735, 2010.

[21] A. Sözen, O. Isikan, T. Menlik, and E. Arcaklioglu, "The forecasting of net electricity consumption of the consumer groups in Turkey," Energy Sources B: Economics, Planning and Policy, vol. 6, no. 1, pp. 20-46, 2011.

[22] G. E. Nasr, E. A. Badr, and M. R. Younes, "Neural networks in forecasting electrical energy consumption: univariate and multivariate approaches," International Journal of Energy Research, vol. 26, no. 1, pp. 67-78, 2002.

[23] A. Azadeh, S. F. Ghaderi, and S. Sohrabkhani, "Annual electricity consumption forecasting by neural network in high energy consuming industrial sectors," Energy Conversion and Management, vol. 49, no. 8, pp. 2272-2278, 2008. 
[24] Z. Guo, W. Zhao, H. Lu, and J. Wang, "Multi-step forecasting for wind speed using a modified EMD-based artificial neural network model," Renewable Energy, vol. 37, no. 1, pp. 241-249, 2012.

[25] V. H. Hinojosa and A. Hoese, "Short-term load forecasting using fuzzy inductive reasoning and evolutionary algorithms," IEEE Transactions on Power Systems, vol. 25, no. 1, pp. 565-574, 2010.

[26] J. Deng, The Primary Methods of Grey System Theory, Huazhong University of Science and Technology Press, Wuhan, China, 2005.

[27] D. Ju-Long, "Control problem of Grey systems," Systems \& Control Letters, vol. 1, no. 5, pp. 288-294, 1982.

[28] T. Zhang, J. J. Cao, X. X. Tie et al., "Water-soluble ions in atmospheric aerosols measured in Xi'an, China: seasonal variations and sources," Atmospheric Research, vol. 102, no. 1-2, pp. 110-119, 2011.

[29] W. Zhou and J.-M. He, "Generalized GM $(1,1)$ model and its application in forecasting of fuel production," Applied Mathematical Modelling, vol. 37, no. 9, pp. 6234-6243, 2013.

[30] L. Wu, S. Liu, L. Yao, and S. Yan, "The effect of sample size on the Grey system model," Applied Mathematical Modelling, vol. 37, no. 9, pp. 6577-6583, 2013.

[31] Y. Wang, X. Chen, Y. Han, and S. Guo, "Forecast of passenger and freight traffic volume based on elasticity coefficient method and Grey model," Procedia-Social and Behavioral Sciences, vol. 96, pp. 136-147, 2013.

[32] M. Meng, D. Niu, and W. Shang, "A small-sample hybrid model for forecasting energy-related CO2 emissions," Energy, vol. 64, pp. 673-677, 2013.

[33] W. Ma, X. Zhu, and M. Wang, "Forecasting iron ore import and consumption of China using Grey model optimized by particle swarm optimization algorithm," Resources Policy, vol. 38, no. 4, pp. 613-620, 2013.

[34] R. B. Carmona Benítez, R. B. Carmona Paredes, G. Lodewijks, and J. L. Nabais, "Damp trend Grey model forecasting method for airline industry," Expert Systems With Applications, vol. 40, no. 12, pp. 4915-4921, 2013.

[35] A. Zolghadri and F. Cazaurang, "Adaptive nonlinear state-space modelling for the prediction of daily mean PM10 concentrations," Environmental Modelling and Software, vol. 21, no. 6, pp. 885-894, 2006.

[36] R. Rajabioun, "Cuckoo optimization algorithm," Applied Soft Computing Journal, vol. 11, no. 8, pp. 5508-5518, 2011.

[37] J. Zhou, R. Fang, Y. Li, Y. Zhang, and B. Peng, "Parameter optimization of nonlinear Grey Bernoulli model using particle swarm optimization," Applied Mathematics and Computation, vol. 207, no. 2, pp. 292-299, 2009.

[38] H. Y. Zhuan, "Mechanism and application of $\mathrm{GM}(1,1)$ and improvement methods," Systems Engineering-Theory \& Practice, vol. 2, pp. 56-62, 1993 (Chinese).

[39] S.-C. Chang, H.-C. Lai, and H.-C. Yu, "A variable P value rolling Grey forecasting model for Taiwan semiconductor industry production," Technological Forecasting and Social Change, vol. 72, no. 5, pp. 623-640, 2005.

[40] H. Kaydani and A. Mohebbi, "A comparison study of using optimization algorithms and artificial neural networks for predicting permeability," Journal of Petroleum Science and Engineering, vol. 112, pp. 17-23, 2013.

[41] The Australian Energy Market Operator, http://www.aemo.com .au/.
[42] Q. Wan, Y. Wei, and S. Yang, "Improving prediction of model Gm $(1,1)$ based on class ratio modeling method," in Proceeding of the 2nd International Symposium on Networking and Network Security (ISNNS '10), p. 73, 2010.

[43] W. Zhao, J. Wang, and H. Lu, "Combining forecasts of electricity consumption in China with time-varying weights updated by a high-order Markov chain model," Omega, vol. 45, pp. 80-91, 2014.

[44] D. Bunn and E. D. Farmer, Comparative Models for Electrical Load Forecasting, Wiley, Chichester, UK, 1985. 


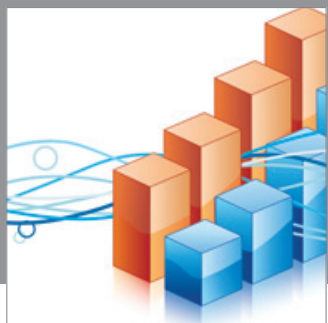

Advances in

Operations Research

mansans

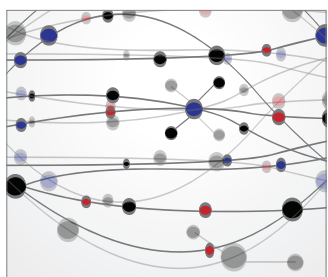

The Scientific World Journal
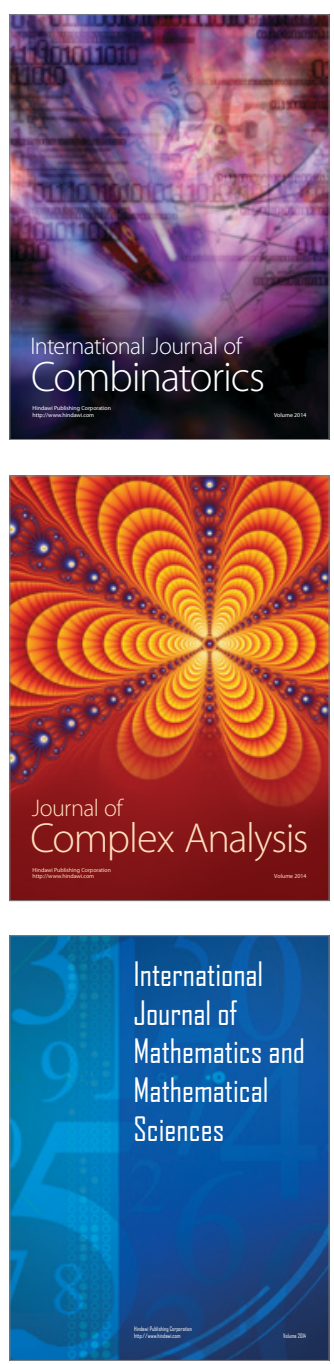
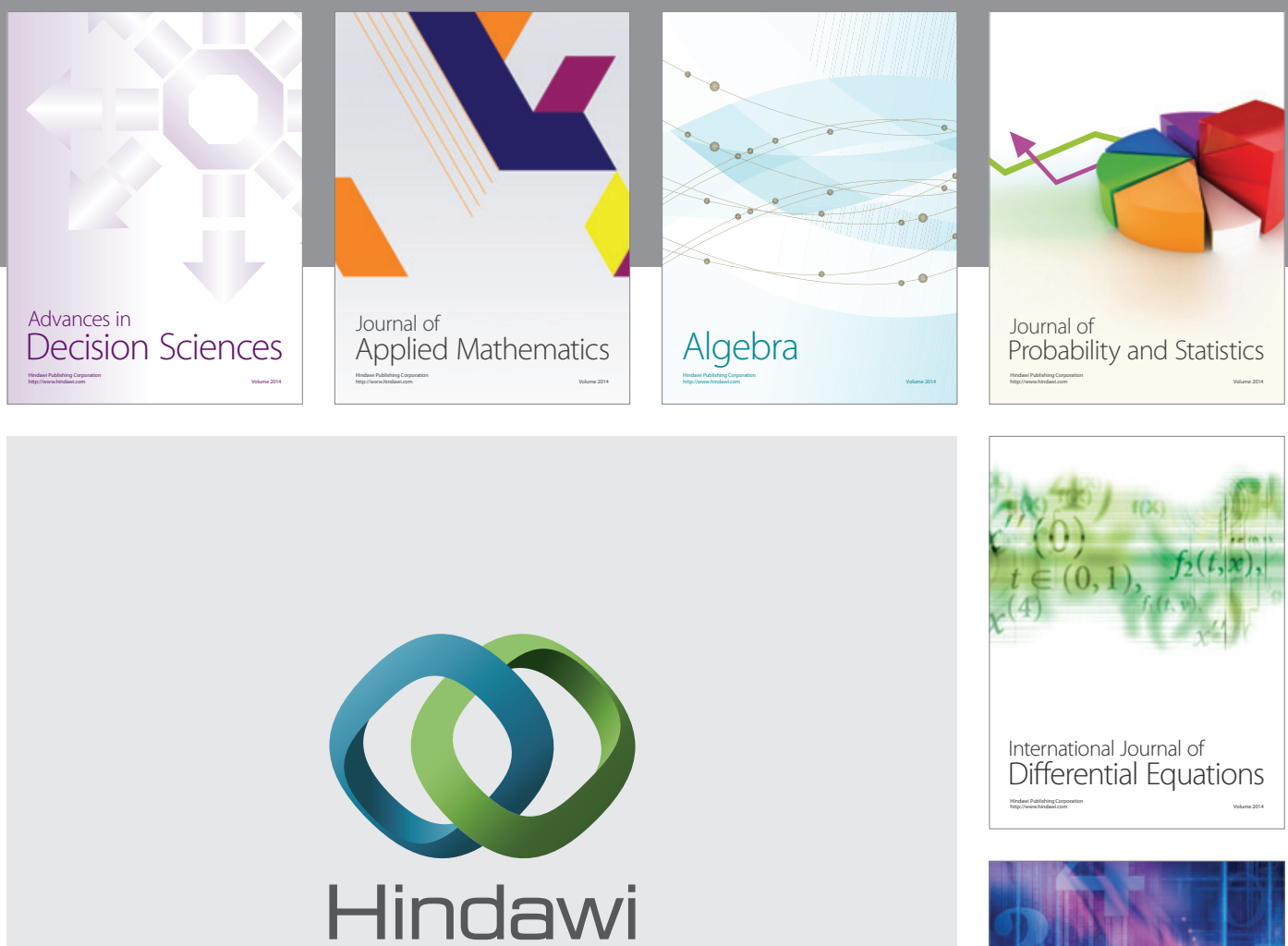

Submit your manuscripts at http://www.hindawi.com
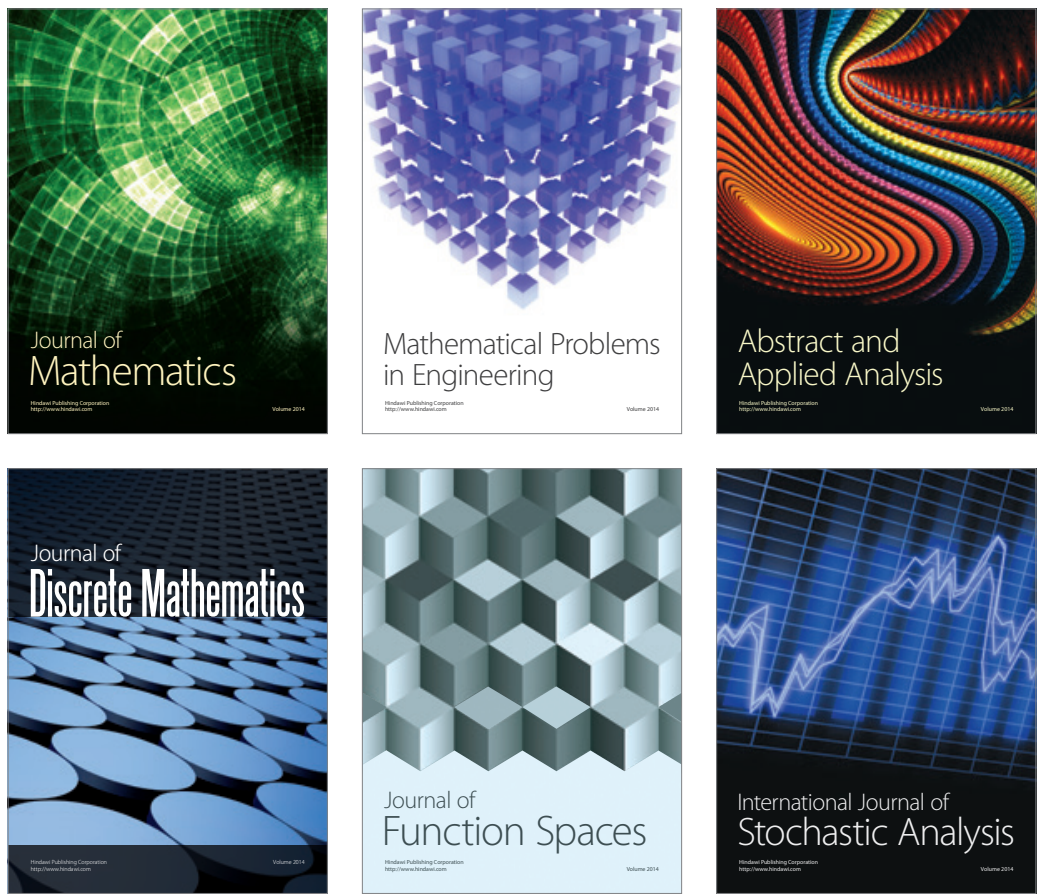

Journal of

Function Spaces

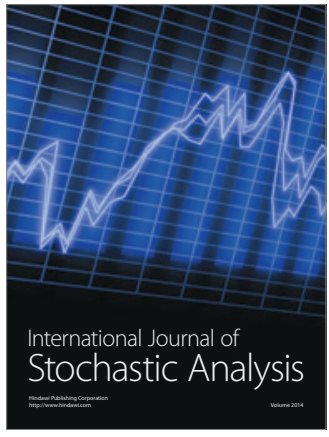

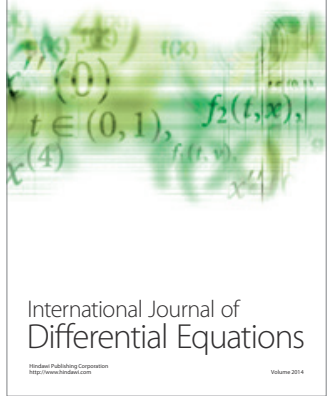
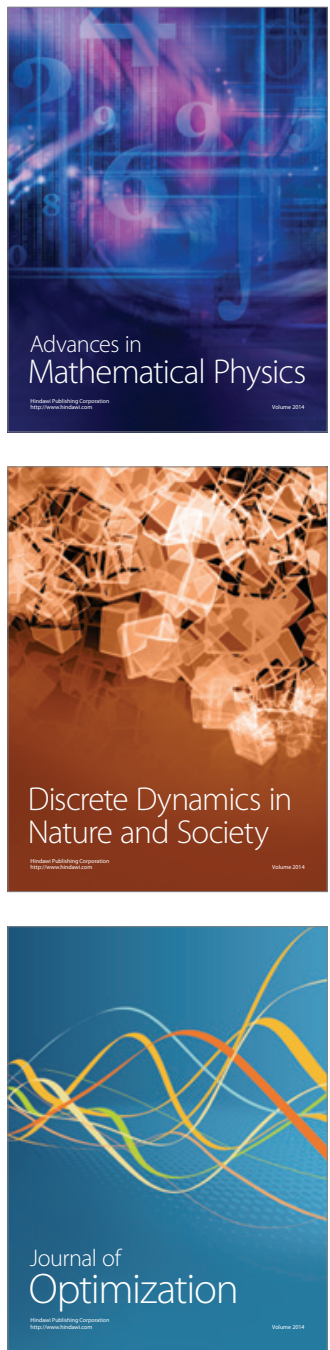